\title{
The nitrogen interaction network in Synechococcus WH5701, a cyanobacterium with two PipX and two $\mathrm{P}_{\mathrm{II}}$-like proteins \\ Correspondence \\ Asunción Contreras \\ contrera@ua.es \\ Received 1 December 2010 \\ Revised 22 December 2010 \\ Accepted 22 December 2010

\author{
Karim Boumediene Laichoubi, ${ }^{1}$ Sabine Beez, ${ }^{2}$ Javier Espinosa, ${ }^{1}$ \\ Karl Forchhammer ${ }^{2}$ and Asunción Contreras ${ }^{1}$ \\ ${ }^{1}$ División de Genética, Universidad de Alicante, Apartado 99, E-03080 Alicante, Spain
${ }^{2}$ Lehrstuhl für Mikrobiologie, Organismische Interaktionen, University Tuebingen, Auf der
Morgenstelle 28, D-72076 Tübingen, Germany
} \\ Nitrogen regulation involves the formation of different types of protein complexes between signal transducers and their transcriptional or metabolic targets. In oxygenic phototrophs, the signal integrator $\mathrm{P}_{\|}$activates the enzyme $\mathrm{N}$-acetyl-L-glutamate kinase (NAGK) by complex formation. $\mathrm{P}_{\|}$ also interacts with $\mathrm{PipX}$, a protein with a tudor-like domain that mediates contacts with $\mathrm{P}_{\|}$and with the transcriptional regulator NtcA, to which it binds to increase its activity. Here, we use a combination of in silico, yeast two-hybrid and in vitro approaches to investigate the nitrogen regulation network of Synechococcus WH5701, a marine cyanobacterium with two $P_{\text {II }}$ (GlnB_A and GInB_B) and two PipX (PipX_I and PipX_II) proteins. Our results indicate that GInB_A is functionally equivalent to the canonical $P_{\|}$protein from Synechococcus elongatus. $G$ InB_A interacted with PipX and NAGK proteins and stimulated NAGK activity, counteracting arginine inhibition. GInB_B had only a slight stimulatory effect on NAGK activity, but its potential to bind effectors and form heterotrimers in Synechococcus WH5701 indicates additional regulatory functions. PipX_II, and less evidently PipX_I, specifically interacted with GInB_A and NtcA, supporting a role for both Synechococcus WH5701 PipX proteins in partner swapping with GlnB_A and NtcA.
}

\section{INTRODUCTION}

Cyanobacteria are phototrophic organisms that perform oxygenic photosynthesis. Autotrophic growth requires the constant assimilation of ammonium via the GS-GOGAT cycle, resulting in consumption of 2-oxoglutarate (MuroPastor et al., 2005). Due to the lack of 2-oxoglutarate dehydrogenase in cyanobacteria, synthesis of 2-oxoglutarate represents the final step in the oxidative branch of the TCA cycle and directly links 2-oxoglutarate levels to nitrogen assimilation (Muro-Pastor et al., 2001). Thus, 2-oxoglutarate accumulates during nitrogen starvation, making this metabolite an excellent indicator of the intracellular carbon to nitrogen balance (Forchhammer, 2004; Laurent et al., 2005). In line with this, interactions of the nitrogen regulators $\mathrm{P}_{\mathrm{II}}$ and NtcA are modulated by this metabolite.

The trimeric $\mathrm{P}_{\text {II }}$ protein is one of the most conserved and widespread signal transduction proteins in nature and plays key roles in nitrogen assimilatory processes (Leigh \&

Abbreviations: FC1/2, flow cell 1/2; NAGK, N-acetyl-L-glutamate kinase; SPR, surface plasmon resonance.

A supplementary table of primer sequences is available with the online version of this paper.
Dodsworth, 2007). $\mathrm{P}_{\mathrm{II}}$ proteins contain three binding sites (one per subunit) for 2-oxoglutarate and ATP (Fokina et al., 2010a) and their primary function is to regulate, by direct protein-protein interactions, the activity of proteins implicated in nitrogen metabolism (reviewed by Forchhammer, 2008). In cyanobacteria, several proteins are known to form complexes with $\mathrm{P}_{\mathrm{II}}$. The first two $\mathrm{P}_{\mathrm{II}}$ receptors were identified in Synechococcus elongatus: the enzyme $N$-acetyl-L-glutamate kinase (NAGK), a $\mathrm{P}_{\mathrm{II}}$ target conserved across domains of life during the evolution of oxygenic photosynthetic organisms (Burillo et al., 2004; Chen et al., 2006; Sugiyama et al., 2004), and the regulatory factor PipX (Burillo et al., 2004; Espinosa et al., 2006). The $\mathrm{P}_{\mathrm{II}}$-NAGK complex consists of a hexameric (trimer of a dimer) NAGK toroid with each of its two faces binding one $\mathrm{P}_{\mathrm{II}}$ trimer along its threefold symmetry axis. In the complex, the T-loops of $\mathrm{P}_{\mathrm{II}}$ are retracted and contact the $\mathrm{N}$ - and C-terminal domain of each enzyme subunit, favouring the catalytically active NAGK conformation (Fokina et al., 2010b; Llácer et al., 2007). In the PipX-P II complexes, one $\mathrm{P}_{\mathrm{II}}$ trimer with its T-loops in a vertically extended conformation sequesters three PipX molecules (Llácer et al., 2010; Zhao et al., 2010). ATP in concert with elevated 2-oxoglutarate levels prevents complex formation 
of $\mathrm{P}_{\mathrm{II}}$ with either NAGK or PipX (Espinosa et al., 2006; Maheswaran et al., 2004), suggesting that PipX-P complexes could also have a function under nitrogen sufficient conditions. Genetic analyses indicate that PipX is toxic to $S$. elongatus in the absence of $\mathrm{P}_{\mathrm{II}}$ (Espinosa et al., 2009, 2010), but the molecular basis of this toxicity remains unknown.

In cyanobacteria, multiple metabolic and developmental processes are induced by nitrogen starvation. NtcA, the global regulator for nitrogen control, activates genes involved in nitrogen assimilation, heterocyst differentiation and acclimation to nitrogen starvation (Herrero et al., 2001; Luque et al., 2001; Sauer et al., 2000). 2-Oxoglutarate, the signal of nitrogen deficiency, stimulates binding of NtcA to target sites (Vázquez-Bermúdez et al., 2002), transcription activation in vitro (Tanigawa et al., 2002) and complex formation between NtcA and PipX (Burillo et al., 2004; Espinosa et al., 2006). The interaction between PipX and NtcA is known to be relevant for maximal activation of NtcA-dependent genes in $S$. elongatus under nitrogen limitation (Espinosa et al., 2006, 2007). The PipX-NtcA complexes consist of one active (2oxoglutarate-bound) NtcA dimer and two PipX molecules. Each NtcA subunit binds one PipX molecule in such a way that it stabilizes the active NtcA conformation (Llácer et al., 2010).

The presence of $\mathrm{P}_{\mathrm{II}}$ and PipX paralogues in some cyanobacteria raises questions on their potential to increase the regulatory capabilities of cells subjected to complex and changing environments. In this context, we use in silico, yeast two-hybrid and in vitro approaches to investigate the nitrogen regulation network of Synechococcus WH5701, a marine cyanobacterium encoding two $\mathrm{P}_{\mathrm{II}}$ and two PipX proteins that thrives in estuaries (Scanlan et al., 2009). The aim was to elucidate interaction partners and particular functions of the $\mathrm{P}_{\mathrm{II}}$ and $\mathrm{PipX}$ proteins encoded by the genome of Synechococcus WH5701.

\section{METHODS}

Strains and growth conditions. Strains and plasmids used in this work are listed in Table 1. Oligonucleotides are described in Supplementary Table S1 (available with the online version of this paper). Constructs were analysed by automated dideoxy DNA sequencing. All cloning procedures were carried out in Escherichia coli DH5 $\alpha$ using standard techniques.

Yeast culture and transformation procedures were as described by Ausubel et al. (1999). Interaction signals between pairs of fusion proteins were determined using the three reporters present in PJ696/ Y187 diploids as described previously (Burillo et al., 2004).

Construction of plasmids. To obtain pUAGC781 and pUAGC786, the $n t c A$ sequence (annotated as ORF WH5701_01395) was amplified by PCR with primers YTH-WHNTCA-F and YTH-WHNTCA-R from Synechococcus WH5701 genomic DNA and cloned into EcoRISalI-digested pGAD424 and pGBT9, respectively.

The $g \ln B A$ (ORF WH5701_15331) and $g \ln B B$ (ORF WH5701_11829) sequences were amplified by PCR using genomic DNA as template with primer pairs YTH-WHGLNB1-F/YTH-WHGLNB1-R and YTHWHGLNB2-F/YTH-WHGLNB2-R, respectively, and cloned into SmaI-BamHI-digested pGAD424 and pGBT9, yielding plasmids pUAGC782, pUAGC787 (GlnB_A:AD and GlnB_A:BD) and pUAGC783 and pUAGC788 (GlnB_B:AD and GlnB_B:BD).

To obtain pUAGC784 and pUAGC789, the pipXI (ORF WH5701_ 00105) sequence was amplified by PCR from genomic DNA with primers YTH-WHPIPX1-F and YTH-WHPIPX1-R and cloned into EcoRI-SalI-digested pGAD424 and pGBT9, respectively. Similarly, the pipXII (ORF WH5701_06246) sequence was amplified with primers YTH-WHPIPX2L-F and YTH-WHPIPX2L-R and cloned into EcoRIBamHI-digested pGAD424 and pGBT9, resulting in plasmids pUAGC785 and pUAGC795.

To obtain pUAGC798 and pUAGC799, the $\operatorname{argB}$ (ORF WH5701_ 12044) sequence was amplified by PCR with primers YTHWHNAGK-F and YTH-WHNAGK-R from genomic DNA of Synechococcus WH5701 and cloned into SalI-BamHI-digested pGAD424 and pGBT9, respectively.

All pGAD derivatives were sequenced using ACTAseq plus GAD-REV primers, and GBT-1F plus GBT-2R primers for pGBT derivatives.

In silico analysis. The sequences of $\mathrm{P}_{\mathrm{II}}$, PipX, NAGK and NtcA homologues from Synechococcus WH5701 were retrieved from the NCBI database. Routinely, alignments were carried out by using ClUSTAL_X 2 (Larkin et al., 2007) with default settings. Crystal complex structures of NAGK- $\mathrm{P}_{\mathrm{II}}, \mathrm{P}_{\mathrm{II}}-\mathrm{PipX}$ and NtcA-PipX were obtained from the protein database (PDB) with accession nos $2 \mathrm{~V} 5 \mathrm{H}$, 2XG8 and 2XKO, respectively. PyMOL 0.99rc6 (DeLano Scientific LLC) was used to represent interaction surfaces of PipX, $\mathrm{P}_{\text {II }}$ and NtcA.

Cloning, overexpression and purification of Synechococcus WH5701 NAGK. To clone argB from Synechococcus sp. WH5701, the gene was amplified by PCR with chromosomal Synechococcus sp. WH5701 DNA and primers NAGKwh5701for and NAGKwh5701rev. The PCR product was first cloned into the A/T cloning site of cloning vector pGEM-T (Promega). The resulting plasmid was restricted with $\mathrm{NdeI}$ and BamHI to isolate the $\operatorname{argB}$-encoding DNA fragment that was then cloned into the NdeI/BamHI site of pET-15b (Novagen), resulting in a gene product with an N-terminally fused $\mathrm{His}_{6}$-tag. To overexpress the recombinant WH5701 $\operatorname{argB}$ gene for purification of NAGK (WH NAGK), the pET15b-WH5701 argB vector was transformed into E. coli BL21(DE3). Overexpression and purification was carried out as described previously (Maheswaran et al., 2004).

Cloning, overexpression and purification of Synechococcus WH5701 GInB_A and GInB_B. WH5701 $g \ln B A$ and $g \ln B B$ genes were amplified by PCR from WH5701 genomic DNA using the following pairs of primers: GlnB1for and GlnB1rev for $g \ln B A$ and GlnB2for and GlnB2rev for $g \ln B \_B$. The resulting PCR products were cloned into the $B s a \mathrm{I}$ site of pASK-IBA3, to yield $g \ln B A$ or $g \ln B B$ products with a C-terminally fused Strep-tag II peptide. The plasmids, pIBA3-GlnB1 and pIBA3-GlnB2, were transformed into E. coli strain BL21(DE3). Overexpression and Strep-tag II affinity purification of the recombinant proteins was carried out as described previously (Heinrich et al., 2004).

NAGK activity assays. The activity of recombinant $\mathrm{His}_{6}$-tagged $\mathrm{WH}$ NAGK was assayed with a coupled enzyme assay, coupling the ADPgenerating NAGK reaction via pyruvate kinase and lactate dehydrogenase to the oxidation of NADH, which was recorded at $340 \mathrm{~nm}$. The assay procedure has been described in detail previously (Beez et al., 2009). One unit of NAGK catalyses the conversion of $1 \mu \mathrm{mol} \mathrm{N}$ acetyl-L-glutamate (NAG) $\mathrm{min}^{-1}$. 
Table 1. Strains used in this work

\begin{tabular}{|c|c|c|}
\hline Strain or plasmid & Genotype or relevant characteristic & Source or reference \\
\hline E. coli DH5 $\alpha$ & $\begin{array}{l}\mathrm{F}^{-} \text {} \phi 80 \mathrm{~d} l a c Z \Delta \mathrm{M} 15 \Delta(\text { lacZYA-argF }) \mathrm{U} 169 \text { endA1 recA1 hsdR17 }\left(\mathrm{r}_{\mathrm{K}}^{-} \mathrm{m}_{\mathrm{K}}^{+}\right) \operatorname{deo} R \\
\text { thi-1 supE44 gyrA96 relA1 } \lambda^{-}\end{array}$ & Hanahan (1985) \\
\hline E. coli $\mathrm{BL} 21(\mathrm{DE} 3)$ & $\begin{array}{l}\mathrm{F}^{-} \text {ompT gal dcm lon } h s d S_{\mathrm{B}}\left(\mathrm{r}_{\mathrm{B}}^{-} \mathrm{m}_{\mathrm{B}}^{-}\right) \lambda[\mathrm{DE} 3 \text { (lacI lacUV5-T7 gene } 1 \text { ind1 sam } 7 \\
\text { nin5)] }\end{array}$ & Phillips et al. (1984) \\
\hline Saccharomyces cerevisiae Y187 & 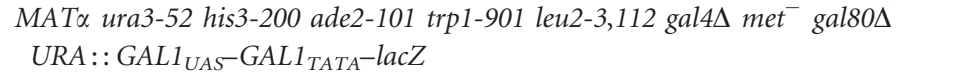 & Harper et al. (1993) \\
\hline Saccharomyces cerevisiae PJ696 & 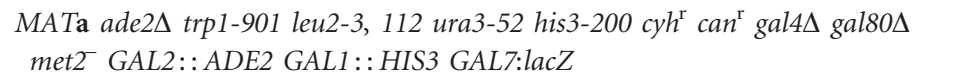 & James et al. (1996) \\
\hline Synechococcus sp. WH5701 & Wild-type Synechococcus sp. WH5701 & D. Scanlan (DNA source) \\
\hline pGAD424 & LEU2, GAL4(768-881)AD, Ap ${ }^{\mathrm{r}}$ & Bartel et al. (1993) \\
\hline pGBT9 & TRP1, GAL4(1-147)BD, Ap ${ }^{\mathrm{r}}$ & Bartel et al. (1993) \\
\hline pUAGC6 & GAL4AD: NtcA (S. elongatus) & Espinosa et al. (2010) \\
\hline pUAGC8 & GAL4BD : NtcA (S. elongatus) & Espinosa et al. (2010) \\
\hline pUAGC11 & GAL4AD : $\mathrm{P}_{\mathrm{II}}$ & Burillo et al. (2004) \\
\hline pUAGC12 & GAL4BD : $\mathrm{P}_{\mathrm{II}}$ & Burillo et al. (2004) \\
\hline pUAGC61 & GAL4AD : NAGK (S. elongatus) & Burillo et al. (2004) \\
\hline pUAGC62 & GAL4BD : NAGK (S. elongatus) & Burillo et al. (2004) \\
\hline pUAGC471 & GAL4AD : PipX & Espinosa et al. (2010) \\
\hline pUAGC472 & GAL4BD: PipX & Espinosa et al. (2010) \\
\hline pUAGC781 & GAL4AD : NtcA (Synechococcus sp.WH5701) & This work \\
\hline pUAGC782 & GAL4AD : GlnB_A & This work \\
\hline pUAGC783 & GAL4AD : GlnB_B & This work \\
\hline pUAGC784 & GAL4AD : PipX_I & This work \\
\hline pUAGC785 & GAL4AD : PipX_II & This work \\
\hline pUAGC786 & GAL4BD : NtcA (Synechococcus sp.WH5701) & This work \\
\hline pUAGC787 & GAL4BD : GlnB_A & This work \\
\hline pUAGC788 & GAL4BD:GlnB_B & This work \\
\hline pUAGC789 & GAL4BD : PipX_I & This work \\
\hline pUAGC795 & GAL4BD : PipX_II & This work \\
\hline pUAGC798 & GAL4AD : NAGK (Synechococcus sp.WH5701) & This work \\
\hline pUAGC799 & GAL4BD : NAGK (Synechococcus sp.WH5701) & This work \\
\hline pASK-IBA3 & $\mathrm{Ap}^{\mathrm{r}}$, StrepTag cloning vector & IBA GmbH \\
\hline pIBA3-GlnB1 & pASK-IBA3 : GlnB_A & This work \\
\hline pIBA3-GlnB2 & pASK-IBA3 : GlnB_B & This work \\
\hline pGEM-T & $\mathrm{Ap}^{\mathrm{r}}$, cloning vector & Promega \\
\hline pGEM-T-WH5701argB & pGEM-T derivative carrying $\arg B$ gene & This work \\
\hline pET-15b & $\mathrm{Ap}^{\mathrm{r}}$, HisTag cloning vector & Novagen \\
\hline pET15b-WH5701argB & pET-15b derivative with $\mathrm{His}_{6}-\mathrm{NAGK}$ & This work \\
\hline
\end{tabular}

Surface plasmon resonance (SPR) experiments. SPR assays were performed using a BIAcore X biosensor system (GE Healthcare). Activation of the NTA-sensor chip with $\mathrm{NiSO}_{4}$ was performed as described previously (Beez et al., 2009). Recombinant $\mathrm{His}_{6}$-tag WH NAGK was loaded on the surface of flow cell 2 (FC2) by injecting $40 \mathrm{nM}$ protein solution in HBS buffer (10 mM Na-HEPES, pH 7.5, $150 \mathrm{mM} \mathrm{NaCl}$ and $0.005 \%$ Nonidet P40) containing $0.5 \mathrm{mM}$ arginine at a flow rate of $10 \mu \mathrm{min}^{-1}$, until a binding signal of approximately 3600 resonance units (RU) had been obtained. To test the binding of GlnB proteins to the FC2 surface-bound NAGK, $25 \mu 1$ of a $1 \mu \mathrm{M}$ solution of GlnB_A or GlnB_B in HBS buffer was injected over flow cell 1 (FC1) and FC2 at a flow rate of $15 \mu \mathrm{min}^{-1}$. The response difference between $\mathrm{FC1}$ and $\mathrm{FC} 2$ was recorded $(\Delta \mathrm{RU})$. Following injection, the sensor chip was washed with HBS buffer to record complex dissociation. To regenerate the surface, NAGK was first removed with $20 \mu \mathrm{l} 1 \mathrm{M}$ imidazole $\mathrm{pH} 7.5$ and after washing with HBS buffer, new NAGK protein was bound as described above.

\section{RESULTS AND DISCUSSION}

\section{Cyanobacteria with a second pipX gene also have a second and atypical $g \ln B$ gene}

The genomes from the cyanobacteria Gloeobacter violaceus, Acaryochloris marina and Synechococcus WH5701 were found to contain two pipX ORFs, while the genomes of other cyanobacteria contained just one. In all three cases, the two PipX predicted sequences were remarkably similar to each other, suggesting independent and relatively recent gene duplication events. The identity of these proteins to $S$. elongatus PipX ranged from 44 to $50 \%$.

Because PipX forms complexes with $\mathrm{P}_{\mathrm{II}}$ and NtcA, and in $S$. elongatus $\mathrm{P}_{\mathrm{II}}$ counteracts PipX toxicity (Espinosa et al., 
2009,2010 ), we wondered if the presence of two pipX genes is associated with more than one gene copy for PipX interacting partners. In all three species, G. violaceus, A. marina and Synechococcus WH5701, a second $g \ln B$-like gene sequence was detected. Only one type of $n t c A$ and $\arg B$ sequence was present in these cyanobacteria.

It has been noted that cyanobacterial $\mathrm{P}_{\mathrm{II}}$ proteins are monophyletic and that a minority of species encode two glnB genes (Sant'Anna et al., 2009; Scanlan et al., 2009). From each of the three genomes with two pipX-like and two $g \ln B$-like genes, one of the two predicted $\mathrm{P}_{\mathrm{II}}$ amino acid sequences, referred to as GlnB_A, was closer to most cyanobacterial $\mathrm{P}_{\text {II }}$ proteins $(83,86$ and $77 \%$ identity was found between S. elongatus and G. violaceus, A. marina and Synechococcus WH5701, respectively). The other sequence, GlnB_B, was not so typical (57, 48 and $65 \%$ identity, respectively, with $S$. elongatus $\mathrm{P}_{\mathrm{II}}$ ), strongly suggesting that GlnB_B proteins were not the result of recent duplication events.

Sequence comparisons suggest that both GlnB_A and GlnB_B proteins may be functional and have a role in carbon to nitrogen balance and energy signalling in these organisms. Most of the key residues conforming to the ATP or 2-oxoglutarate binding sites are present in both GlnB_A and GlnB_B. There is one K40R change in the 2oxoglutarate binding site of Gloeobacter GlnB_B and two changes in Acaryochloris GlnB_B, one in the ATP binding site (R101Q) and one in the 2-oxoglutarate binding site (K40Q). Furthermore, most of the residues involved in intersubunit interactions are also conserved between $S$. elongatus $\mathrm{P}_{\mathrm{II}}$ and $\mathrm{G} \ln \mathrm{B} \_\mathrm{A}$ or $\mathrm{Gln} \mathrm{B} \_\mathrm{B}$, the few exceptions being conservative changes or changes affecting regions of higher variability in cyanobacteria (Table 2 and data not shown). Thus, the conservation and similarity of trimerization surfaces between both types of proteins suggests the possibility of heterotrimer formation in vivo.

The differences between typical and atypical $\mathrm{P}_{\text {II }}$ proteins from these three cyanobacteria are particularly evident at the region expanding the T-loop, where most of the residues forming the cyanobacterial signature (Palinska et al., 2002) are nested. Except for an I18V change present in Synechococcus WH5701, all 14 signature residues were present in the three GlnB_A proteins, while 7, 8 or up to 12 changes were present in their GlnB_B counterparts (Table 3 ), thus strongly supporting the idea that GlnB_A proteins are the orthologues of $\mathrm{P}_{\mathrm{II}}$ and that functions involving $\mathrm{T}$ loop interactions are likely to be defective in GlnB_B proteins. The R45-S49-E85 NAGK interaction signature (Llácer et al., 2007) is part of the cyanobacterial $\mathrm{P}_{\mathrm{II}}$ signature. Residue R45 is absent in all three GlnB_B proteins, with $\mathrm{S} 49$ and E85 absent in two of them, strongly suggesting that GlnB_A, but not GlnB_B proteins, would be involved in regulation of NAGK activity in these three cyanobacteria.

\section{Structure-informed prediction of interacting partners in the nitrogen regulation network of Synechococcus WH5701}

As a first approach to elucidate the regulatory connections that could take place in cyanobacteria encoding two $\mathrm{P}_{\mathrm{II}}$ and two PipX proteins, we focused on one of these organisms, Synechococcus WH5701. Its two predicted pipX products were named as PipX_I and PipX_II. We disregarded the wrong start codon annotated in the database for PipX_II and used instead the previous AUG that seems the most likely start codon. The inclusion of 25 additional amino acids at the $\mathrm{N}$ terminus of PipX_II considerably increased its similarity to other PipX proteins, given that this region of the tudor domain is important for PipX-mediated interactions and is therefore conserved.

The information gained from the structurally characterized NAGK- $\mathrm{P}_{\text {II }}$, PipX-P $\mathrm{P}_{\text {II }}$ and PipX-NtcA complexes of $S$. elongatus was next used to find out the most likely interacting partners for Synechococcus WH5701. To facilitate comparison between interaction surfaces of homologue proteins, residues differing in Synechococcus WH5701 proteins are also shown in Fig. 1.

Only one conservative change (E15D) in the $\mathrm{P}_{\mathrm{II}}$ surface interacting with NAGK was found in GlnB_A (the D15 residue is also found in GlnB_B) (Fig. 1a). This similarity

Table 2. Amino acid substitution residues involved in $P_{\|}$intersubunit contacts present in $G \ln B \_A$ and $G \ln B \_B$

Substitutions are indicated for each position (when present) in amino acid letter code. For simplicity, the nine residues that do not change are not shown.

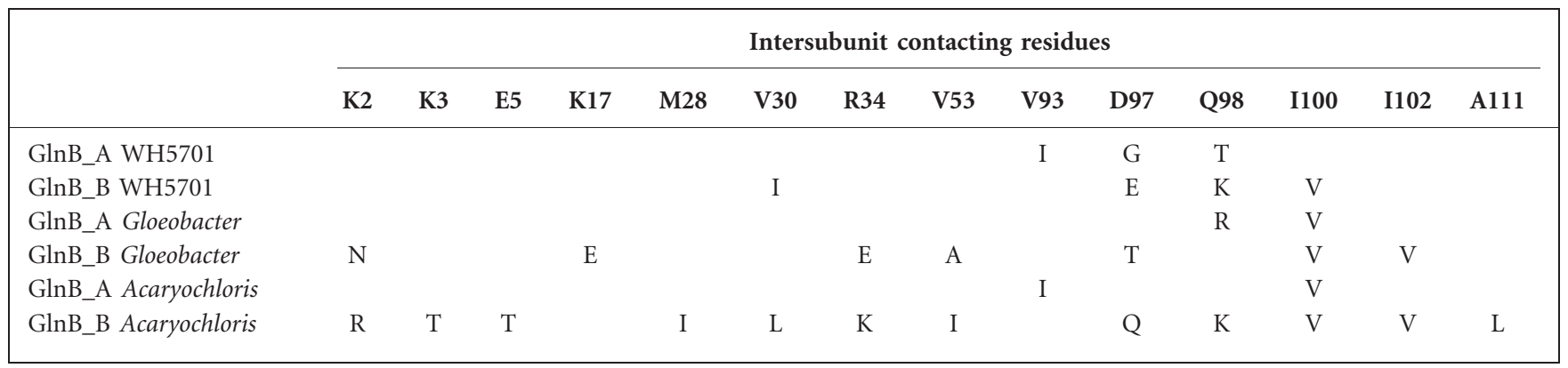


Table 3. Amino acid substitution of residues that constitute the cyanobacterial $P_{\|}$signature

Substitutions are indicated for each position (when present) in amino acid letter code. There were no substitutions in Synechococcus $\mathrm{P}_{\mathrm{II}}$, Gloeobacter GlnB_A or Acaryochloris GlnB_A.

\begin{tabular}{|c|c|c|c|c|c|c|c|c|c|c|c|c|c|c|}
\hline & \multicolumn{14}{|c|}{ Cyanobacterial $P_{I I}$ signature } \\
\hline & R9 & I18 & I/V26 & S31 & R34 & Q42 & R45 & S49 & T52 & E54 & Q57 & L59 & E85 & S94 \\
\hline GlnB_A WH5701 & & $\mathrm{V}$ & & & & & & & & & & & & \\
\hline GlnB_B WH5701 & & $\mathrm{T}$ & & $\mathrm{T}$ & & & $\mathrm{T}$ & $\mathrm{A}$ & & & $\mathrm{N}$ & $\mathrm{V}$ & & $\mathrm{T}$ \\
\hline GlnB_B Gloeobacter & Q & Q & Q & & $\mathrm{E}$ & $\mathrm{H}$ & I & $\mathrm{F}$ & A & Q & A & & $\mathrm{S}$ & $\mathrm{L}$ \\
\hline GlnB_B Acaryochloris & & $\mathrm{L}$ & & & $\mathrm{K}$ & $\mathrm{S}$ & Q & & $\mathrm{E}$ & & $\mathrm{R}$ & & Q & \\
\hline
\end{tabular}

strongly argues in favour of very similar functions for $S$. elongatus $\mathrm{P}_{\mathrm{II}}$ and $\mathrm{GlnB}$ _A. Three additional substitutions, F11V and two changes affecting two out of the three NAGK interaction signature residues (R45T and S49A), were present at GlnB_B, arguing against conservation of function. Thus, these sequence features and the similarity of GlnB_A and NAGK from Synechococcus WH5701 (WH NAGK) to their homologues in $S$. elongatus agrees with previous predictions of conservation of the $\mathrm{P}_{\mathrm{II}}-\mathrm{NAGK}$ interaction in organisms that perform oxygenic photosynthesis (Burillo et al., 2004). In addition, the data also suggest the possibility of cross-regulation between heterologous proteins.

GlnB_A was more similar to $\mathrm{P}_{\mathrm{II}}$ than $\mathrm{G} \ln \mathrm{B} \_\mathrm{B}$ with respect to the $\mathrm{P}_{\mathrm{II}}$ surface interactions with PipX (Fig. 1b). Two conservative changes were found in GlnB_A, Y51F (a change also present in $\mathrm{GlnB}$ _B and other $\mathrm{P}_{\mathrm{II}}$ proteins from cyanobacteria and plants) and D14E, whose proximity to D15 (E15 in S. elongatus) restored the Glu-Asp pair (AspGlu in S. elongatus). In addition to the above-mentioned F11V change, three other differences (E32D, Q57N and L59V) were found in GlnB_B. Although it is difficult to predict the impact of these differences on interactions with the PipX_I and PipX_II proteins, it seems reasonable to argue that GlnB_A would be a better binding partner than GlnB_B for (at least) S. elongatus PipX.

In agreement with the great similarity between the two Synechococcus WH5701 PipX proteins, most of the differences compared with $S$. elongatus PipX at the interaction surface of the tudor domain were found in both PipX_II and PipX_I (Fig. 1c-d), with only one conservative change (L14M) specific to PipX_I. Four substitutions were identical (L36M, P51V, G53P and F42L) and one was found in two versions (R54L/R54Y). Thus, the possibility that both PipX_II and PipX_I would be able to form complexes with GlnB_A could not be excluded at this stage. Given the similarity between the different proteins and the architecture of the $\mathrm{P}_{\mathrm{II}}-\mathrm{PipX}$ complexes, heterologous interactions also seem likely $\left(\mathrm{P}_{\mathrm{II}^{-}}\right.$ PipX_II, PII-PipX_I, GlnB_A-PipX).

Since the PipX regions interacting with $\mathrm{P}_{\mathrm{II}}$ and NtcA overlap, the rationale just discussed for GlnB_A applies to putative PipX interactions with NtcA. Except for F42, which did not contact NtcA, all substitutions discussed for
PipX also affect residues involved in direct contacts with NtcA, a protein with $71 \%$ identity to that of S. elongatus. Differences at two of the nine residues making direct contacts with PipX were found for Synechococcus WH5701 NtcA (WH NtcA), corresponding to the substitutions S218A and Q219K (Fig. 1e), which are also common in the cyanobacteria group. It seems reasonable to assume that this NtcA protein could interact with PipX_II and PipX_I.

\section{Protein interactions involving nitrogen regulators from Synechococcus WH5701}

Previously we used yeast two-hybrid approaches to infer the existence of $\mathrm{P}_{\mathrm{II}}-\mathrm{NAGK}, \mathrm{P}_{\mathrm{II}}-\mathrm{PipX}$ and PipX-NtcA complexes in $S$. elongatus and to provide evidence of the specificity of the interactions between wild-type and mutant derivatives of the different proteins involved (Burillo et al., 2004; Espinosa et al., 2006, 2010; Llácer et al., 2007, 2010). Here, we generated additional twohybrid constructs to analyse interactions between the relevant proteins from Synechococcus WH5701. These include NAGK, NtcA, the two $\mathrm{P}_{\mathrm{II}}(\mathrm{GlnB} B \mathrm{~A}, \mathrm{GlnB} B \mathrm{~B})$ and the two PipX paralogues (PipX_II and PipX_I). Expression of reporters was determined in Y187/PJ696 diploids as described previously (Burillo et al., 2004). In all cases, proteins were fused independently to both GAL4BD and GAL4AD domains. Fusion proteins from the model cyanobacterium $S$. elongatus were included in the analyses, allowing us to obtain information for heterologous recognition between $S$. elongatus and Synechococcus WH5701 proteins. A typical yeast two-hybrid interaction assay for the Synechococcus WH5701 proteins is shown in Fig. 2(a). Results from pairing of heterologous proteins, alongside already known interactions for $S$. elongatus proteins, are summarized in Fig. 2(b).

As predicted in the light of the extensive residue conservation between $\mathrm{G} \ln \mathrm{B} \_\mathrm{A}$ and $\mathrm{P}_{\mathrm{II}}$, particularly at the interacting surface, strong interaction signals were found between WH NAGK and GlnB_A. Consistent with the lack of key residues at $\mathrm{G} \ln \mathrm{B} \_\mathrm{B}$, no interaction signal could be detected between WH NAGK and GlnB_B. GlnB_A, but not GlnB_B, interacted with all three PipX proteins, strongly suggesting that $\mathrm{GlnB} B \mathrm{~B}$ is not able to perform the two functions that have been characterized for $\mathrm{P}_{\mathrm{II}}$ in 


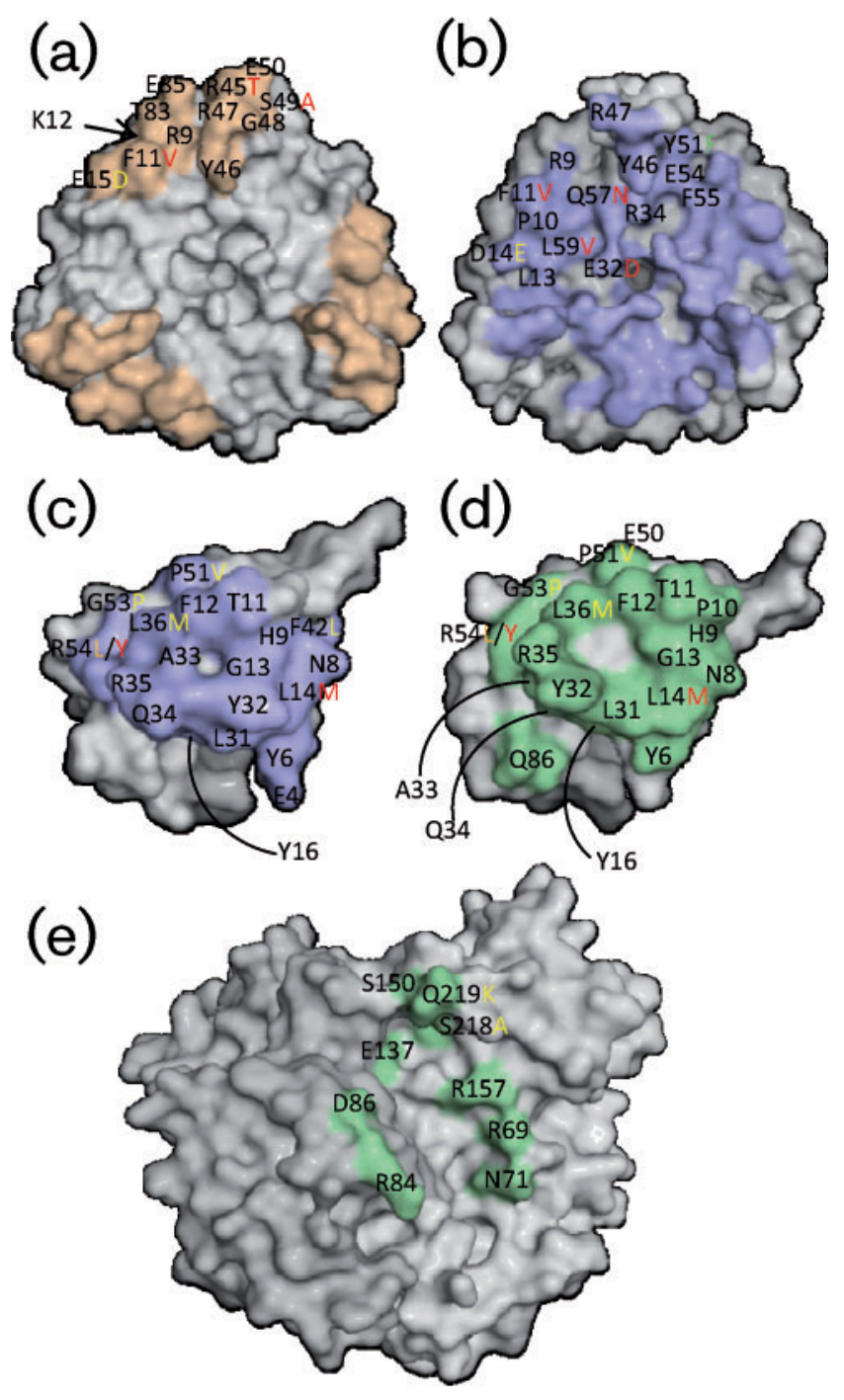

Fig. 1. Relevant residues in Synechococcus WH5701 proteins compared with those involved in $\mathrm{P}_{\| I}-\mathrm{NAGK}, \mathrm{P}_{\| I}-\mathrm{PipX}$ and NtcAPipX complexes from S. elongatus. (a) Surface representation of $\mathrm{P}_{\|}$in the $\mathrm{P}_{\| \mid}-\mathrm{NAGK}$ complex (PDB file $2 \mathrm{~V} 5 \mathrm{H}$ ) with the interaction surface in light brown. Substitutions on key interacting residues (for one $P_{\|}$subunit) present in $G \ln B \_A, G \ln B$ B $B$ or both, are indicated in yellow, red and green, respectively. (b) Surface representation of $P_{\| \mid}$in the $P_{\|}-$PipX complex (PDB file 2XG8) with the interaction surface in blue. Substitutions are indicated as in (a). (c, d) Surface representation of PipX in the $P_{\|}-$PipX (PDB file 2XKO) and NtcA-PipX (2XG8) complexes coloured in blue and green, respectively. Substitutions on key interacting residues present in PipX_II, PipX_I or both are indicated in orange, red and yellow, respectively. (e) Surface representation of the NtcA interaction surface, coloured green, in the NtcA-PipX complex (PDB file 2XKO). Key residues are numbered and substitutions on Synechococcus WH5701 NtcA are shown in yellow.

cyanobacteria, that is NAGK regulation and sequestration of PipX. All three PipX derivatives interacted with S. elongatus $\mathrm{P}_{\mathrm{II}}$ and $\mathrm{Gln} \mathrm{B} \_\mathrm{A}$, and signals from some of the heterologous pairs (such as $\mathrm{P}_{\mathrm{II}} / \mathrm{PipX}$ _II) were as strong as those between the S. elongatus $\left(\mathrm{P}_{\mathrm{II}} / \mathrm{PipX}\right)$ or Synechococcus WH5701 proteins (GlnB_A/PipX_II). Both GlnB_A and GlnB_B interacted with themselves, which agrees with their predicted trimeric structure, and with each other (also to $S$. elongatus $\mathrm{P}_{\mathrm{II}}$ ), which supports the possibility that they form heterotrimers in vivo. If that was the case, the presence of GlnB_B in Synechococcus WH5701 could interfere with the formation of complexes by GlnB_A. This would be reminiscent of the situation in other bacteria for which heterotrimer formation for $\mathrm{P}_{\mathrm{II}}$ proteins ( $\mathrm{GlnK}$ and $\mathrm{GlnB}$ in E. coli) have been demonstrated (Forchhammer et al., 1999).

Interestingly, although the PipX proteins from Synechococcus WH5701 were closer to each other than to S. elongatus PipX (Fig. 1c, d), interaction signals with the NtcA proteins from each of the two organisms were clearly detected for $S$. elongatus PipX and PipX_II, but hardly for PipX_I. Since the only relevant difference between PipX_II and PipX_I at the interacting surface affects positions 14 and 54 (Fig. 1b-c), one or two of the Leu residues present in PipX_II (Y54 and M14 in PipX_I) may be responsible for the stronger interaction signals between PipX_II and NtcA. Differences at these residues could also explain the lower interaction signals of PipX_I with $\mathrm{P}_{\mathrm{II}}$ and GlnB_A, compared with those of PipX_II.

Detection of signals between PipX_I and NtcA proteins (and here Synechococcus WH5701 and S. elongatus NtcA proteins were indistinguishable) required longer incubation times and was possible just for the BD:PipX_I/ $\mathrm{AD}$ : NtcA pairs. However, signals were independently detected on both selective media and X-Gal indicator plates, and were significantly higher than those from the negative controls (Fig. $2 \mathrm{~b}$ and data not shown). We are inclined to think that the extremely weak interaction signals observed for BD : PipX_I/AD : NtcA fusion proteins could be relevant, and therefore the possibility of complex formation between NtcA and PipX_I in Synechococcus WH5701 should not be excluded at present.

Taken together, the yeast two-hybrid data point to PipX_II as a true PipX orthologue involved in partner swapping with GlnB_A and NtcA, but do not necessarily exclude the PipX_INtcA interaction in Synechococcus WH5701. The weaker interaction signals mediated by PipX_I, in comparison with PipX_II, could be interpreted in evolutionary terms as a sign of divergence in which one of the duplicated proteins (PipX_I) would tend to lose some of its functions, in this case the ability to perform partner swapping with $\mathrm{P}_{\mathrm{II}}$ and NtcA. It would be interesting to know whether this apparent loss of activity is accompanied by acquisition of new functions.

\section{In vitro properties of NAGK-P |I complexes from Synechococcus WH5701 and activation of NAGK proteins by GInB_A and GInB_B}

Subsequent biochemical analysis of complexes involving Synechococcus WH5701 proteins was performed using 

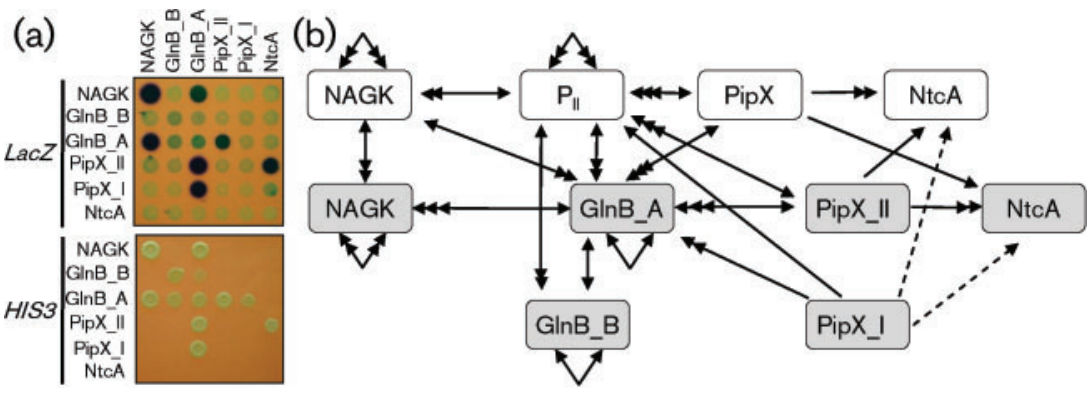

Fig. 2. Yeast two-hybrid interactions mediated by $P_{l l}$, NAGK, PipX and NtcA from Synechococcus WH5701. (a) Representative interaction assay for Synechococcus WH5701 proteins. (b) Schematic representation of yeast two-hybrid interactions amongst nitrogen regulators from S. elongatus and Synechococcus WH5701 proteins (white and grey boxes, respectively). The number of arrowheads indicates the relative strength of the interaction as described by Burillo et al. (2004). Dashed lines indicate very weak signals (PipX_I-NtcA).

purified NAGK, GlnB_A and GlnB_B, essentially as described previously for the S. elongatus proteins (Heinrich et al., 2004; Maheswaran et al., 2004). The difficulty of obtaining soluble proteins for Synechococcus WH5701 NtcA, PipX_II and PipX_I precluded analysis of the corresponding complexes at this stage, and therefore we focused on the properties of the different $\mathrm{P}_{\mathrm{II}}-\mathrm{NAGK}$ complexes.

First, we used purified proteins to independently assay the physical interaction between NAGK and GlnB_A or GlnB_B by SPR spectroscopy analyses. As shown in Fig. 3, rapid complex formation on the WH NAGK surface was observed with GlnB_A, but no interaction could be detected with GlnB_B. Therefore, the results from this in vitro analysis closely matched those obtained with the yeast-two hybrid system.

Next we determined the enzymic activity of Synechococcus WH NAGK in the absence and presence of GlnB_A or GlnB_B proteins. As shown in Fig. 4(a), WH NAGK is very inactive in the absence of a $\mathrm{P}_{\text {II }}$ protein. The free enzyme does not show Michaelis-Menten kinetics, but instead a slow sigmoidal increase of activity with increasing substrate. Below $15 \mathrm{mM}$ NAG, almost no activity could be detected; the activity could only be reasonably assayed at $50 \mathrm{mM} \mathrm{NAG} \mathrm{(data} \mathrm{not} \mathrm{shown).}$ Addition of GlnB_A dramatically increased the activity of WH NAGK and resulted in a typical MichaelisMenten kinetics with an apparent $K_{\mathrm{m}}$ for NAG of $1.5 \pm 0.5 \mathrm{mM}$ and a $V_{\max }$ of $7.3 \pm 0.6 \mathrm{U} \mathrm{mg}^{-1}$. Notably, addition of GlnB_B (but not BSA when used as a control; data not shown) also had a slight, but measurable, stimulatory effect on NAGK activity, resulting in a hyperbolic substrate saturation curve with an apparent $K_{\mathrm{m}}$ for NAG of $4.9 \pm 1.2 \mathrm{mM}$ and a $V_{\max }$ of $1.9 \pm 0.2 \mathrm{U}$ $\mathrm{mg}^{-1}$.

As expected for a typical hexameric NAGK, the WH NAGK activity was subjected to non-competitive inhibition by arginine. In the absence of $\mathrm{P}_{\text {II }}$ proteins, the half-maximal inhibitory concentration $\left(\mathrm{IC}_{50}\right)$ was approximately $40 \mu \mathrm{M}$. Addition of $\mathrm{Gln} B$ _A reduced the inhibitory effect of arginine on enzyme activity by 40 fold, shifting the $\mathrm{IC}_{50}$ to $1.6 \mathrm{mM}$ (Fig. $4 \mathrm{~b}$ ). In contrast,

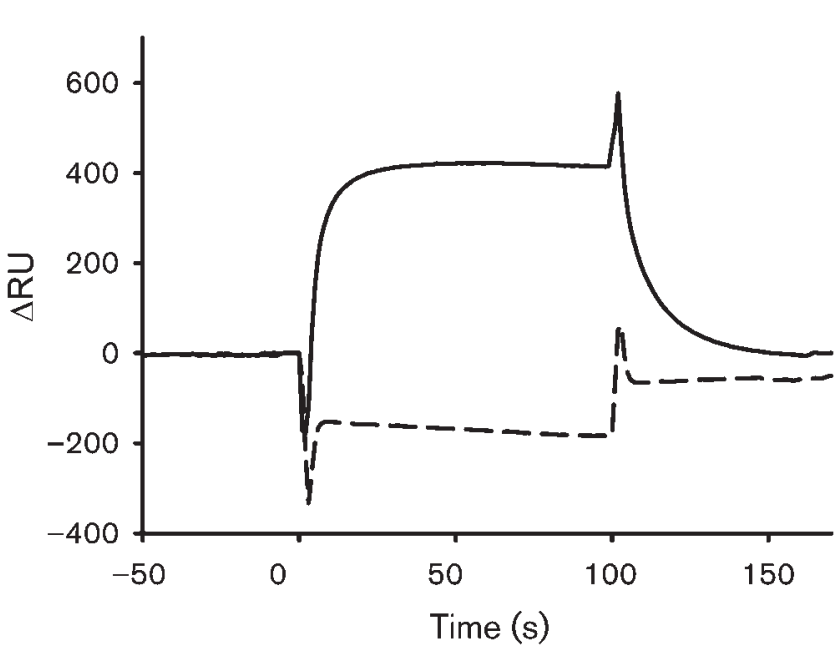

Fig. 3. BiaCore SPR analysis of complex formation of WH NAGK with GlnB_A or GlnB_B. The sensor chip was prepared by bringing approximately 3600 resonance units (RU) $\mathrm{His}_{6}$-tagged WH NAGK to the surface of FC2 of an NTA-sensor chip, with FC1 serving as a control. To assay the interaction with $\mathrm{G} \ln B$ proteins, $25 \mu \mathrm{l}$ of a $1 \mu \mathrm{M}$ GlnB_A (solid line) or GlnB_B (dashed line) solution were injected as analyte at a flow rate of $15 \mu \mathrm{min}^{-1}$ over $F C 1$ and $F C 2$, and the response difference $(\triangle R U)$ between $F C 1$ and FC2 was recorded. Following injection, the surfaces of FC1 and FC2 were washed with HBS buffer (see Methods).

GlnB_B has almost no effect on arginine inhibition (Fig. $4 \mathrm{~b}$, inset).

In summary, we have demonstrated that GlnB_A is able to significantly stimulate NAGK activity by changing its kinetic properties and decreasing the sensitivity to arginine inhibition. In addition to unravelling molecular details of the predicted interaction between WH NAGK and GlnB_A, we show that GlnB_B is still able to weakly stimulate WH NAGK in vitro. The latter result, which is at odds with sequence predictions and the negative results obtained with two solid techniques for protein-protein interactions (Figs 2 and 3), suggest that the GlnB_B-WH NAGK interaction responsible for the weak stimulation of NAGK activity 

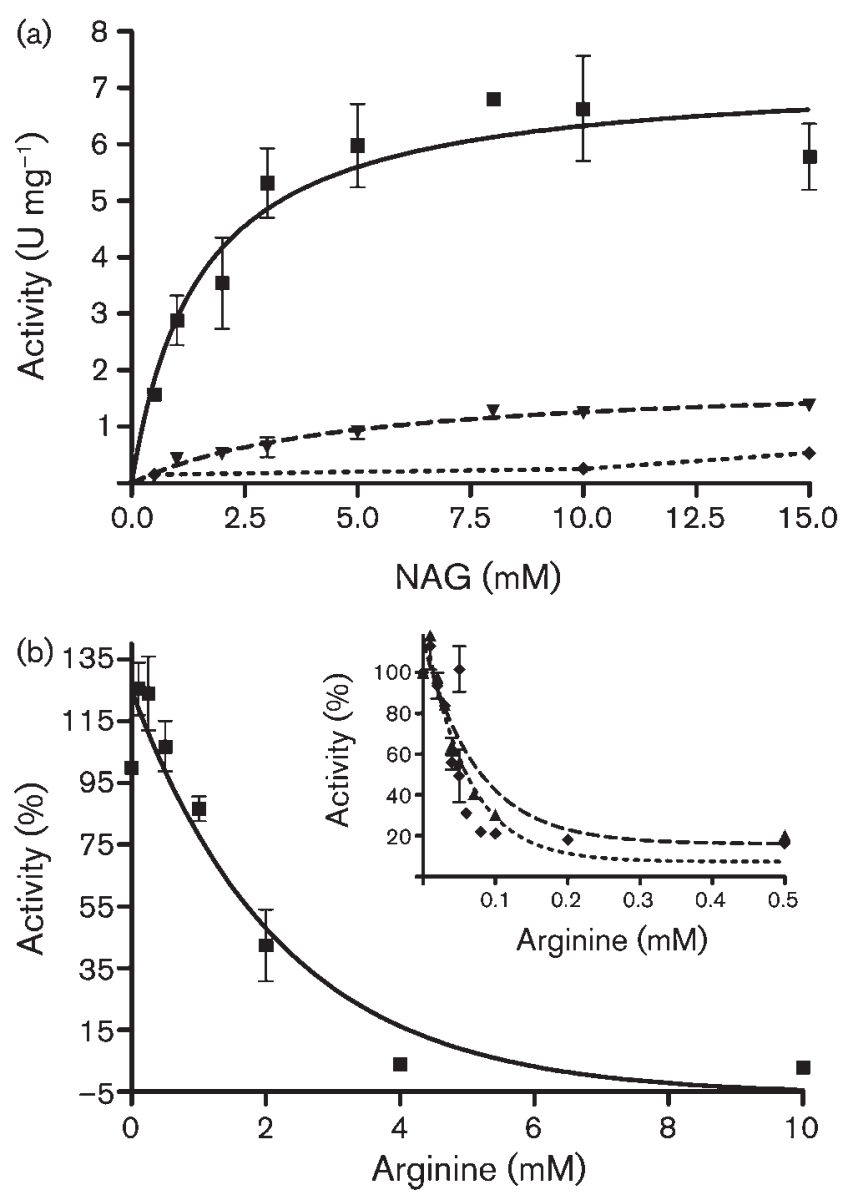

Fig. 4. Influence of $G \ln B \_A$ and $G \ln B$ B on WH5701 NAGK activity. (a) NAGK activity assays were performed using $3 \mu \mathrm{g}$ recombinant $\mathrm{WH}$ NAGK in the absence ( $\boldsymbol{\nabla}$, dotted line) or presence of $1.2 \mu \mathrm{g} G \ln B \_A(\boldsymbol{\square}$, solid line) or $\mathrm{G} \operatorname{lnB} B \mathrm{~B}$ ( $\boldsymbol{\nabla}$, dashed line) as described in Methods. The mean $( \pm S D)$ values of triplicate assays are shown. (b) Inhibition of WH NAGK activity by arginine in the absence or presence of GInB_A or GInB_B. Coupled assays were performed in the presence of $50 \mathrm{mM} \mathrm{NAG}$ and $10 \mathrm{mM}$ ATP together with increasing concentrations of arginine, as indicated. Assays using $3 \mu \mathrm{g}$ NAGK and $1.2 \mu \mathrm{g}$ GlnB_A or GlnB_B were performed as described in Methods. In the presence of GInB_A ( $\boldsymbol{\square}$, solid line) the half-maximal inhibitory concentration of arginine is approximately $1.6 \mathrm{mM}$, whereas the free WH NAGK (see inset, $\checkmark$ and dotted line) shows half maximal inhibition at approximately $40 \mu \mathrm{M}$ arginine. Addition of GlnB_B (inset, $\boldsymbol{\Delta}$ and dashed line) has almost no effect on allosteric arginine inhibition.

observed in vitro is too weak and transient for detection by yeast two-hybrid or SPR assays. Whatever the case, the possibility of heterotrimerization also calls attention to the regulatory potential associated with the presence of two $\mathrm{P}_{\mathrm{II}}$ proteins in Synechococcus WH5701.

\section{Concluding remarks}

This work brings attention to the regulatory potential of cyanobacteria such as $G$. violaceus, $A$. marina and
Synechococcus WH5701 encoding paralogues of $\mathrm{P}_{\mathrm{II}}$ and PipX, two proteins involved in nitrogen signal transduction by protein-protein interactions. We have experimentally addressed basic questions on the nitrogen interaction network of Synechococcus WH5701, an euryhaline cyanobacterium encoding each two $\mathrm{P}_{\mathrm{II}}$ (GlnB_A and GlnB_B) and PipX (PipX_II and PipX_I) proteins. Our results show that GlnB_A and PipX_II are functionally equivalent to $\mathrm{P}_{\mathrm{II}}$ and PipX from the model cyanobacterium S. elongatus. PipX_II, and to a lesser extent PipX_I, specifically interacted with GlnB_A and NtcA, in agreement with the idea that at least PipX_II would mediate partner swapping between GlnB_A and NtcA according to the intracellular 2-oxoglutarate levels. GlnB_A significantly stimulates NAGK activity and antagonizes inhibition by arginine. Given the potential of $G \ln B$ B to form heterotrimers with $G \ln B \_A$, it is tempting to propose that in Synechococcus WH5701, GlnB_B could act as a negative regulator to fine-tune the efficiency of GlnB_A. The slight activation of NAGK by GlnB_B could allow a fine-tuned activation of the enzyme depending on its degree of heterotrimerization with GlnB_A. Further work is required to understand the molecular details and particularly the possible contributions of this complicated nitrogen interaction network to the adaptations of Synechococcus WH5701 to the wide range of salinity and nutrient conditions arising in estuaries.

\section{ACKNOWLEDGEMENTS}

This work was supported by grants from Ministerio de Ciencia e Innovación (BFU2009-07371) and Ministerio de Educación y Ciencia (HA2007-0074). We thank D. Scanlan for Synechococcus WH5701 DNA and helpful advice, Olexandra Fokina for graphical artwork, and J. L. Llácer and V. Rubio for constructive discussions.

\section{REFERENCES}

Ausubel, F. M., Brent, R., Kingston, R. E. \& Moore, D. D. (editors) (1999). Short Protocols in Molecular Biology: a Compendium of Methods from Current Protocols in Molecular Biology. New York: Wiley.

Bartel, P., Chien, C. T., Sternglanz, R. \& Fields, S. (1993). Using the two hybrid system to detect protein-protein interactions. In Cellular Interactions in Development: A Practical Approach, pp. 153-179. Edited by D. A. Hartley. Oxford, UK: Oxford University Press.

Beez, S., Fokina, O., Herrmann, C. \& Forchhammer, K. (2009). $\mathrm{N}$-acetyl-L-glutamate kinase (NAGK) from oxygenic phototrophs: $\mathrm{P}_{\text {II }}$ signal transduction across domains of life reveals novel insights in NAGK control. J Mol Biol 389, 748-758.

Burillo, S., Luque, I., Fuentes, I. \& Contreras, A. (2004). Interactions between the nitrogen signal transduction protein PII and $N$-acetyl glutamate kinase in organisms that perform oxygenic photosynthesis. J Bacteriol 186, 3346-3354.

Chen, Y. M., Ferrar, T. S., Lohmeier-Vogel, E. M., Morrice, N., Mizuno, Y., Berenger, B., Ng, K. K., Muench, D. G. \& Moorhead, G. B. (2006). The PII signal transduction protein of Arabidopsis thaliana forms an arginine-regulated complex with plastid $\mathrm{N}$-acetyl glutamate kinase. J Biol Chem 281, 5726-5733. 
Espinosa, J., Forchhammer, K., Burillo, S. \& Contreras, A. (2006). Interaction network in cyanobacterial nitrogen regulation: PipX, a protein that interacts in a 2-oxoglutarate dependent manner with PII and NtcA. Mol Microbiol 61, 457-469.

Espinosa, J., Forchhammer, K. \& Contreras, A. (2007). Role of the Synechococcus PCC 7942 nitrogen regulator protein PipX in NtcAcontrolled processes. Microbiology 153, 711-718.

Espinosa, J., Castells, M. A., Laichoubi, K. B. \& Contreras, A. (2009). Mutations at $p i p X$ suppress lethality of PII-deficient mutants of Synechococcus elongatus PCC 7942. J Bacteriol 191, 4863-4869.

Espinosa, J., Castells, M. A., Laichoubi, K. B., Forchhammer, K. \& Contreras, A. (2010). Effects of spontaneous mutations in PipX functions and regulatory complexes on the cyanobacterium Synechococcus elongatus strain PCC 7942. Microbiology 156, 1517-1526.

Fokina, O., Chellamuthu, V. R., Forchhammer, K. \& Zeth, K. (2010a). Mechanism of 2-oxoglutarate signaling by the Synechococcus elongatus PII signal transduction protein. Proc Natl Acad Sci U S A 107, 1976019765.

Fokina, O., Chellamuthu, V. R., Zeth, K. \& Forchhammer, K. (2010b). A novel signal transduction protein $\mathrm{P}_{\mathrm{II}}$ variant from Synechococcus elongatus PCC 7942 indicates a two-step process for NAGK-P $\mathrm{P}_{\text {II }}$ complex formation. J Mol Biol 399, 410-421.

Forchhammer, K. (2004). Global carbon/nitrogen control by PII signal transduction in cyanobacteria: from signals to targets. FEMS Microbiol Rev 28, 319-333.

Forchhammer, K. (2008). $\mathrm{P}_{\text {II }}$ signal transducers: novel functional and structural insights. Trends Microbiol 16, 65-72.

Forchhammer, K., Hedler, A., Strobel, H. \& Weiss, V. (1999). Heterotrimerization of PII-like signalling proteins: implications for PII-mediated signal transduction systems. Mol Microbiol 33, 338-349.

Hanahan, D. (1985). Techniques for transformation of Escherichia coli. In DNA Cloning, pp. 109-135. Edited by D. Glover. Oxford, UK: IRL Press.

Harper, J. W., Adami, G. R., Wei, N., Keyomarsi, K. \& Elledge, S. J. (1993). The p21 Cdk-interacting protein Cip1 is a potent inhibitor of G1 cyclin-dependent kinases. Cell 75, 805-816.

Heinrich, A., Maheswaran, M., Ruppert, U. \& Forchhammer, K. (2004). The Synechococcus elongatus PII signal transduction protein controls arginine synthesis by complex formation with $\mathrm{N}$-acetyl-Lglutamate kinase. Mol Microbiol 52, 1303-1314.

Herrero, A., Muro-Pastor, A. M. \& Flores, E. (2001). Nitrogen control in cyanobacteria. $J$ Bacteriol 183, 411-425.

James, P., Halladay, J. \& Craig, E. A. (1996). Genomic libraries and a host strain designed for highly efficient two-hybrid selection in yeast. Genetics 144, 1425-1436.

Larkin, M. A., Blackshields, G., Brown, N. P., Chenna, R., McGettigan, P. A., McWilliam, H., Valentin, F., Wallace, I. M., Wilm, A. \& other authors (2007). CLUSTAL W and CLUSTAL_X version 2.0. Bioinformatics 23, 2947-2948.

Laurent, S., Chen, H., Bédu, S., Ziarelli, F., Peng, L. \& Zhang, C. C. (2005). Nonmetabolizable analogue of 2-oxoglutarate elicits heterocyst differentiation under repressive conditions in Anabaena sp. PCC 7120. Proc Natl Acad Sci U S A 102, 9907-9912.

Leigh, J. A. \& Dodsworth, J. A. (2007). Nitrogen regulation in bacteria and archaea. Annu Rev Microbiol 61, 349-377.

Llácer, J. L., Contreras, A., Forchhammer, K., Marco-Marín, C., Gil-Ortiz, F., Maldonado, R., Fita, I. \& Rubio, V. (2007). The crystal structure of the complex of PII and acetylglutamate kinase reveals how PII controls the storage of nitrogen as arginine. Proc Natl Acad Sci U S A 104, 17644-17649.

Llácer, J. L., Espinosa, J., Castells, M. A., Contreras, A., Forchhammer, K. \& Rubio, V. (2010). Structural basis for the regulation of NtcA-dependent transcription by proteins PipX and PII. Proc Natl Acad Sci U S A 107, 15397-15402.

Luque, I., Zabulon, G., Contreras, A. \& Houmard, J. (2001). Convergence of two global transcriptional regulators on nitrogen induction of the stress-acclimation gene $n b l A$ in the cyanobacterium Synechococcus sp. PCC 7942. Mol Microbiol 41, 937-947.

Maheswaran, M., Urbanke, C. \& Forchhammer, K. (2004). Complex formation and catalytic activation by the PII signaling protein of $\mathrm{N}$-acetyl-L-glutamate kinase from Synechococcus elongatus strain PCC 7942. J Biol Chem 279, 55202-55210.

Muro-Pastor, M. I., Reyes, J. C. \& Florencio, F. J. (2001). Cyanobacteria perceive nitrogen status by sensing intracellular 2oxoglutarate levels. J Biol Chem 276, 38320-38328.

Muro-Pastor, M. I., Reyes, J. C. \& Florencio, F. J. (2005). Ammonium assimilation in cyanobacteria. Photosynth Res 83, 135-150.

Palinska, K. A., Laloui, W., Bédu, S., Loiseaux-de Goër, S., Castets, A. M., Rippka, R. \& Tandeau de Marsac, N. (2002). The signal transducer $\mathrm{P}_{\mathrm{II}}$ and bicarbonate acquisition in Prochlorococcus marinus PCC 9511, a marine cyanobacterium naturally deficient in nitrate and nitrite assimilation. Microbiology 148, 24052412.

Phillips, T. A., VanBogelen, R. A. \& Neidhardt, F. C. (1984). lon gene product of Escherichia coli is a heat-shock protein. J Bacteriol 159, 283-287.

Sant'Anna, F. H., Trentini, D. B., de Souto Weber, S., Cecagno, R., da Silva, S. C. \& Schrank, I. S. (2009). The PII superfamily revised: a novel group and evolutionary insights. J Mol Evol 68, 322-336.

Sauer, J., Dirmeier, U. \& Forchhammer, K. (2000). The Synechococcus strain PCC $7942 \mathrm{gln} N$ product (glutamine synthetase III) helps recovery from prolonged nitrogen chlorosis. J Bacteriol 182, 56155619.

Scanlan, D. J., Ostrowski, M., Mazard, S., Dufresne, A., Garczarek, L., Hess, W. R., Post, A. F., Hagemann, M., Paulsen, I. \& Partensky, F. (2009). Ecological genomics of marine picocyanobacteria. Microbiol Mol Biol Rev 73, 249-299.

Sugiyama, K., Hayakawa, T., Kudo, T., Ito, T. \& Yamaya, T. (2004). Interaction of $\mathrm{N}$-acetylglutamate kinase with a PII-like protein in rice. Plant Cell Physiol 45, 1768-1778.

Tanigawa, R., Shirokane, M., Maeda Si, S., Omata, T., Tanaka, K. \& Takahashi, H. (2002). Transcriptional activation of NtcA-dependent promoters of Synechococcus sp. PCC 7942 by 2-oxoglutarate in vitro. Proc Natl Acad Sci U S A 99, 4251-4255.

Vázquez-Bermúdez, M. F., Herrero, A. \& Flores, E. (2002). 2-Oxoglutarate increases the binding affinity of the NtcA (nitrogen control) transcription factor for the Synechococcus $g \ln A$ promoter. FEBS Lett 512, 71-74.

Zhao, M. X., Jiang, Y. L., Xu, B. Y., Chen, Y., Zhang, C. C. \& Zhou, C. Z. (2010). Crystal structure of the cyanobacterial signal transduction protein PII in complex with PipX. J Mol Biol 402, 552-559.

Edited by: C.-C. Zhang 